\title{
Molecular phylogeny of the higher and lower taxonomy of the Fusarium genus and differences in the evolutionary histories of multiple genes
}

Maiko Watanabe ${ }^{1 *}$, Takahiro Yonezawa ${ }^{2}$, Ken-ichi Lee ${ }^{3}$, Susumu Kumagai ${ }^{3}$, Yoshiko Sugita-Konishi ${ }^{1}$, Keiichi Goto ${ }^{4}$ and Yukiko Hara-Kudo ${ }^{1}$

\begin{abstract}
Background: Species of the Fusarium genus are important fungi which is associated with health hazards in human and animals. The taxonomy of this genus has been a subject of controversy for many years. Although many researchers have applied molecular phylogenetic analysis to examine the taxonomy of Fusarium species, their phylogenetic relationships remain unclear only few comprehensive phylogenetic analyses of the Fusarium genus and a lack of suitable nucleotides and amino acid substitution rates. A previous stugy with whole genome comparison among Fusairum species revealed the possibility that each gene in Fusarium genomes has a unique evolutionary history, and such gene may bring difficulty to the reconstruction of phylogenetic tree of Fusarium. There is a need not only to check substitution rates of genes but also to perform the exact evaluation of each gene-evolution.

Results: We performed phylogenetic analyses based on the nucleotide sequences of the rDNA cluster region (rDNA cluster), and the $\beta$-tubulin gene $(\beta$-tub), the elongation factor $1 \alpha$ gene $(E F-1 \alpha)$, and the aminoadipate reductase gene (lys2). Although incongruence of the tree topologies between lys 2 and the other genes was detected, all genes supported the classification of Fusarium species into 7 major clades, I to VII. To obtain a reliable phylogeny for Fusarium species, we excluded the lys2 sequences from our dataset, and re-constructed a maximum likelihood (ML) tree based on the combined data of the rDNA cluster, $\beta$-tub, and EF-1 $\alpha$. Our ML tree indicated some interesting relationships in the higher and lower taxa of Fusarium species and related genera. Moreover, we observed a novel evolutionary history of lys2. We suggest that the unique tree topologies of lys2 are not due to an analytical artefact, but due to differences in the evolutionary history of genomes caused by positive selection of particular lineages.
\end{abstract}

Conclusion: This study showed the reliable species tree of the higher and lower taxonomy in the lineage of the Fusarium genus. Our ML tree clearly indicated 7 major clades within the Fusarium genus. Furthermore, this study reported differences in the evolutionary histories among multiple genes within this genus for the first time.

\section{Background}

Species of the Fusarium genus are well-known important plant pathogens, and are mycotoxin producers which are associated with human and animal health hazards [1,2]. Fusarium species are well-researched in many fields, such as molecular biology, ecology, phytopathology, medicalmycology, toxicology, and others. One problem commonly encountered by researchers interested in Fusarium species

\footnotetext{
* Correspondence: mwatanabe@nihs.go.jp

'Division of Microbiology, National Institute of Health Sciences, Kamiyoga 1-

18-1, Setagaya-ku, Tokyo 158-8501, Japan

Full list of author information is available at the end of the article
}

is the precise taxonomic system of the genus. In general, species are recognized on the basis of the morphological species concept, the biological species concept, the phylogenetic species concept or a combination of these [3]. Current classification schemes of fungi are exclusively based on the morphological species concept, and identification of the species primarily involves the use of morphological characteristics [4]. Although traditional taxonomic systems for the Fusarium genus have been proposed based on the morphological species concept, the taxonomy of this genus has been debated for many years [5-10]. Recently, many researchers have applied molecular phylogenetic 
analysis to examine the taxonomy of Fusarium species, and have proposed new taxonomic systems based on the phylogenetic species concept. However, many phylogenetic relationships remain unclear as only few comprehensive phylogenetic analyses of the Fusarium genus have been performed. Moreover, some previous studies have reported phylogenetic trees with a low resolution, especially in the deep lineages, due to a lack of suitable nucleotide and amino acid substitution rates and other factors.

Recently, whole genome comparison among four Fusairum species revealed the drastic genome-evolution such as the horizontal gene transfer in Fusarium genomes [11]. Therefore, it is possible for each gene in Fusarium genomes to have a unique evolutionary history, and it is necessary to perform exact evaluation of the evolutionary processes of each gene. Moreover, a unique evolutionary history of each gene in Fusarium genomes may bring difficulty to the reconstruction of phylogenetic tree among Fusarium species as mentioned above. There is a need not only to check the nucleotide and amino acid substitution rates of genes but also to perform the exact evaluation of each gene-evolution. Then, we should select suitable genes providing information for phylogenetic inference about both the higher and lower taxa in the Fusarium tree.

Previously, some parts of the rDNA cluster region, $\beta$-tub, and $E F-1 \alpha$ have been used as genetic markers for the phylogenetic analysis of fungal taxonomic groups, including Fusarium species [12-14]. Furthermore, it has been reported that the lys 2 may be a good phylogenetic marker for inferring relationships among fungal genera [15] and among species of the Byssochlamys genus and related genera [16]. In this study, we evaluated holistically the rDNA cluster region, $\beta$-tub, EF- $1 \alpha$, and lys 2 as markers to infer the reliable species tree of Fusarium species, and re-constructed the phylogenetic tree using the maximum likelihood method.

\section{Results and discussions}

\section{Seven major clades of the Fusarium genus and the incongruence of gene trees}

The ML trees inferred from each of the concatenated parts of the rRNA cluster region (rDNA cluster), E-tub EF-1 D and lys 2 are displayed in Figures 1, 2, 3 and 4, respectively. The tree topologies of the gene sequences were not consistent with each other. However, all of the gene trees supported the classification of Fusarium species into 7 major clades, namely, clades I to VII. Most of the support values for these clades were very high (more than $95 \%$ bootstrap value; BP), with the exceptions of clade I (71\% BP) and clade II (75\% BP) of the $\beta$-tub ML tree, clade V $(88 \% \mathrm{BP})$ and clade VII $(55 \% \mathrm{BP})$ of the rDNA cluster ML tree, and clade VII $(<50 \%$ BP) of the lys $2 \mathrm{ML}$ tree. Our ML tree clearly indicated 7 major clades within the Fusarium genus for the first time.

Many of taxonomic studies based on morphological characters have reported that some "sections", including closely related species, share some "synapomorphic" character states. Clade I consists of F. larvarum and F. merismoides which belong to different "sections", namely, Eupionnotes and Arachnites, respectively. Although the $\beta$-tub and EF-1 $\alpha$ sequences supported the monophyly of $F$. merismoides, the rDNA cluster supported a paraphyletic relationship for this species. Clades II, III, and IV consist of single species, namely, F. dimerum, F. solani, and F. decemcellulare, respectively. Clade V contains 2 "sections": Elegans, which consists of F. oxysporum, and Liseola, which consists of F. subglutinans, F. proliferatum and F. verticillioides. Clade VI consists of F. lateritium, F. avenaceum, and F. tricinctum, which belong to different "sections", namely, Lateritium, Roseum, and Sporotrichiella respectively. The paraphyly of $F$. avenaceum and F. lateritium was supported by all the genes. Clade VII contains 4 "sections" with 9 species: Eupionnotes consisting of F. incarnatum, Gibbosum consisting of F. equiseti and F. acuminatum, Discolor consisting of F. graminearum and F. culmorum, and Sporotrichiella consisting of F. poae, F. kyusyuense, $F$. sporotrichioides, and F. langsethiae. Our ML trees based on each of the rDNA cluster and the 3 genes indicate that the species in each of the clades (I to VII) have close relationships with each other.

Some taxonomic groups which were not previously identified using the morphological species concept, have already revealed by molecular phylogenetic analyses previously reported. O'Donnell et al. [14] showed that there are species complexes including more than 2 species, such as the Gibberella fujikuroi species complex. O'Donnell et al. [14] and O'Donnell and Cigelnik [14,17] showed that some sections proposed by morphological studies form paraphyletic or polyphyletic groups, such as Sporotrichiella and Discolor. This study supported their results. Moreover, our results indicated some new taxonomic groups, such as clade I and VI. The close relationships in two clades are discussed in the paragraph of "Verification of monophyly of the sections and species of the Fusarium genus", as described below.

\section{Evaluation of incongruence of the gene trees}

The phylogenetic relationships among clades I to VII differ from each other, as described above. To evaluate this incongruence, we compared the log-likelihood scores of the relationships among clades I to VII (Figure 1) (see Additional file 1). The absolute values of the log likelihood scores of the ML trees, and the differences between the log-likelihood scores of the ML tree $\pm 1 \mathrm{SE}$ and of the 


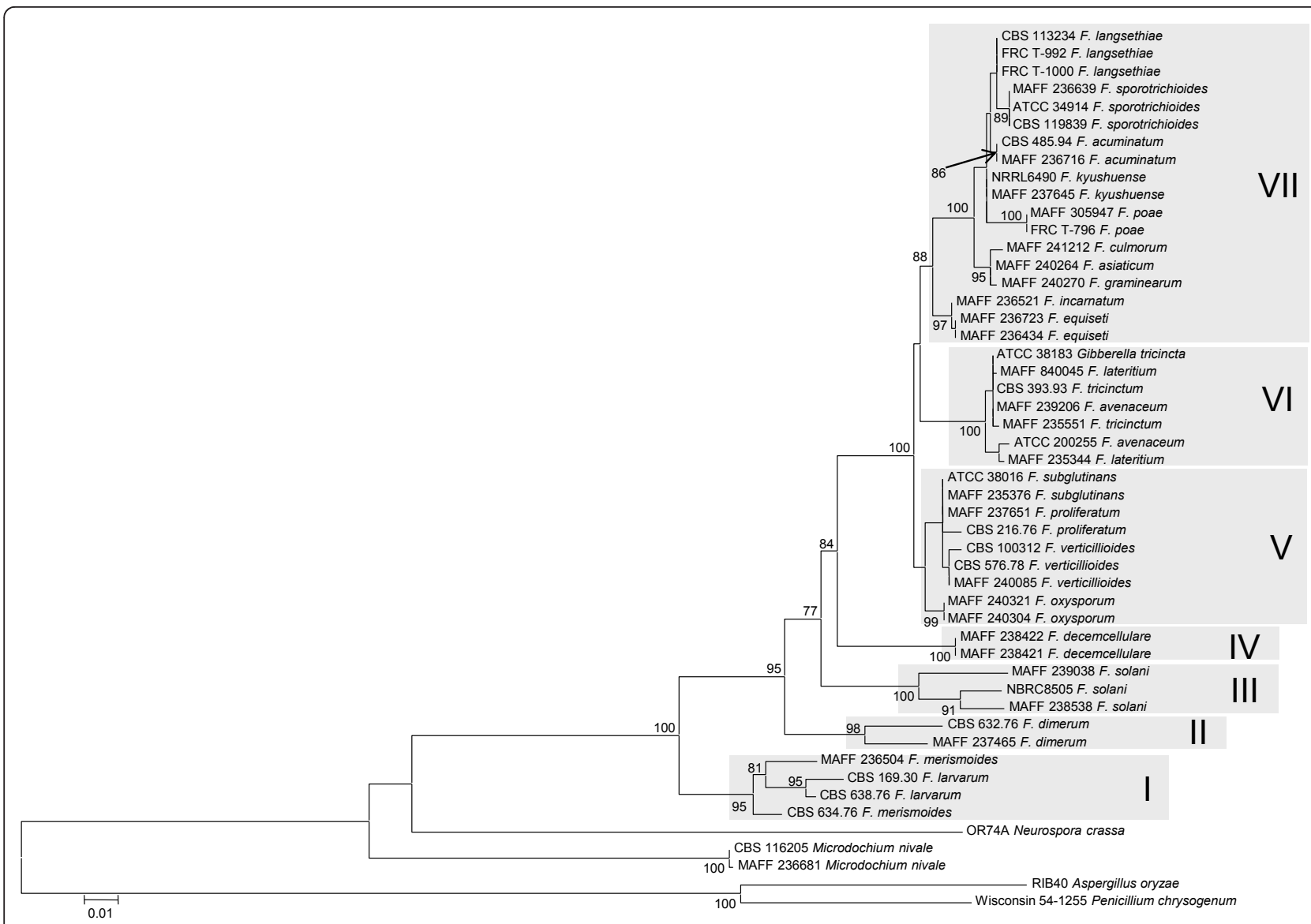

Figure 1 Maximum likelihood trees for the Fusarium genus and related genera inferred from the rDNA cluster including 18S rDNA, ITS1, 5.8S rDNA and 28S rDNA. The GTR $+I+\Gamma$ model was used as the model for nucleotide substitution. Branch lengths are proportional to the estimated number of nucleotide substitutions. The BP values over 75\% are displayed on the nodes (BP; 1000 replicates).

alternative trees are displayed in Table 1 . It can be concluded that. no significant differences were observed between the EF-1 $\alpha$, rDNA cluster, and $\beta$-tub ML trees, but all of these differed from the lys $2 \mathrm{ML}$ tree (p-value of the SH test $<0.001)$.

What is the reason for the differences in tree topology observed among the genes? One possibility is that the tree inference is an artefact. Another possibility is that the genes have different evolutionary histories. In the former case, the main factors which can lead to misleading tree inferences include (1) long-branch attraction, (2) composition bias of nucleotide and amino acid, and (3) convergent evolution.

Long-branch attraction mainly occurs as a consequence of rapidly evolving sites, and removal of such sites from the analysis can reduce the effects of long-branch attraction [18]. Accordingly, we excluded the fast evolving sites of lys2. After the $3^{\text {rd }}$ codon position sites or synonymous substitution sites had been excluded, the tree topology remained essentially the same as the ML tree displayed in
Figure 4. Furthermore, even when the ML tree was inferred based upon the $2^{\text {nd }}$ codon positions only, the tree topology remained essentially the same as the ML tree displayed in Figure 4. Therefore, the possibility of long-branch attraction is unlikely.

Extreme composition bias of nucleotides and amino acids can strongly mislead tree inference $[19,20]$. Therefore, we examined composition bias of lys 2 nucleotides and amino acids in the lineages within our dataset. However, no composition bias was observed in the amino acid sequences or the combined $1^{\text {st }} / 2^{\text {nd }}$ codon position sites. Composition bias of the $3^{\text {rd }}$ codon position sites was detected in F. lateritium (MAFF235344 and MAFF840045), and of the combined $1^{\text {st }}, 2^{\text {nd }}$ and $3^{\text {rd }}$ codon position sites in F. tricinctum (CBS393.93, ATCC38183, and MAFF235551). These species both belong to clade VI. Therefore, it is unlikely that nucleotide composition bias can explain the phylogenetically "misleading" observed among clades I to VII in ML trees displayed in Figures 1, 2, 3 and 4. 


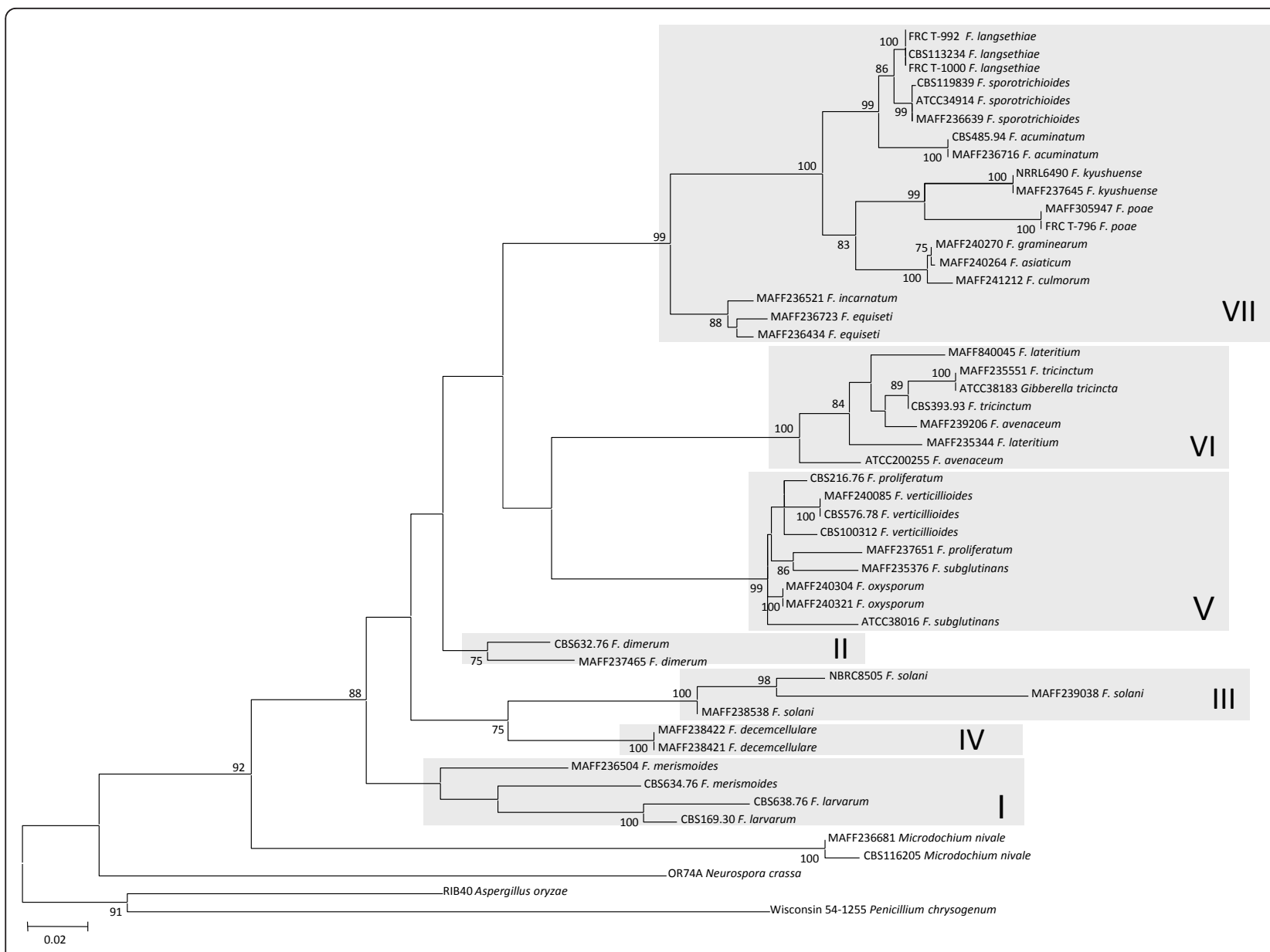

Figure 2 Maximum likelihood trees for the Fusarium genus and related genera inferred from $\boldsymbol{\beta}$-tub. The GTR $+I+\Gamma$ model was used as the model for nucleotide substitution. Branch lengths are proportional to the estimated number of nucleotide substitutions. Each codon position was analysed separately. The BP values over 75\% are displayed on the nodes (BP; 1000 replicates).

Although the principal theory of molecular phylogenetics is based on the neutral theory of molecular evolution [21], convergent evolution is known to occur at the molecular level and can mislead the reconstruction of phylogenetic trees $[22,23]$. As natural selection generally acts at the level of amino acid sequences, synonymous substitutions are unlikely to be affected by convergence. Therefore, we inferred the phylogenetic tree using only the $3^{\text {rd }}$ codon positions because substitutions at these sites are mainly synonymous. However, the lys $2 \mathrm{ML}$ tree inferred only using the $3^{\text {rd }}$ codon positions was essentially the same as the tree presented in Figure 4 (data not displayed).

For these 3 reasons, it is unlikely that the incongruence of the lys $2 \mathrm{ML}$ tree and the other gene trees was due to an analytical artefact. Instead, the differences in tree topology may reflect differences in the evolutionary histories of the considered genes. Therefore, when we reconstructed the species tree, we excluded lys 2 from the analysis.

\section{Evaluation of genetic markers for phylogenetic reconstruction}

To accurately reconstruct the phylogenetic tree, we selected the genes which displayed an adequate evolutionary rate. ML trees based on each individual gene or the rDNA regions are displayed in Figures 2, 3 and 4 and additional files 2, 3, 4 and 5 . The substitution rates of the 3 rDNA regions $(5.8 \mathrm{~S}, 18 \mathrm{~S}$, and $28 \mathrm{~S}$ ) were all slow. Although the substitution rate of ITS1 was faster than that of the rDNA genes, the sequence length was very short $(101 \mathrm{bp})$. Therefore, the nucleotide sequences of each 4 rDNA regions were identical in several Fusarium species. When the 3 rDNA genes and ITS1 were combined in a cluster, we could distinguish most of the species from the nucleotide sequence data. However, since only small differences were observed among species, some resolutions among the species in the same clade were unclear (Figure 1). In contrast, the nucleotide substitution rates of the protein coding genes, namely, $\beta$-tub, EF-1 $\alpha$, and lys2, were rapid, and each of these 


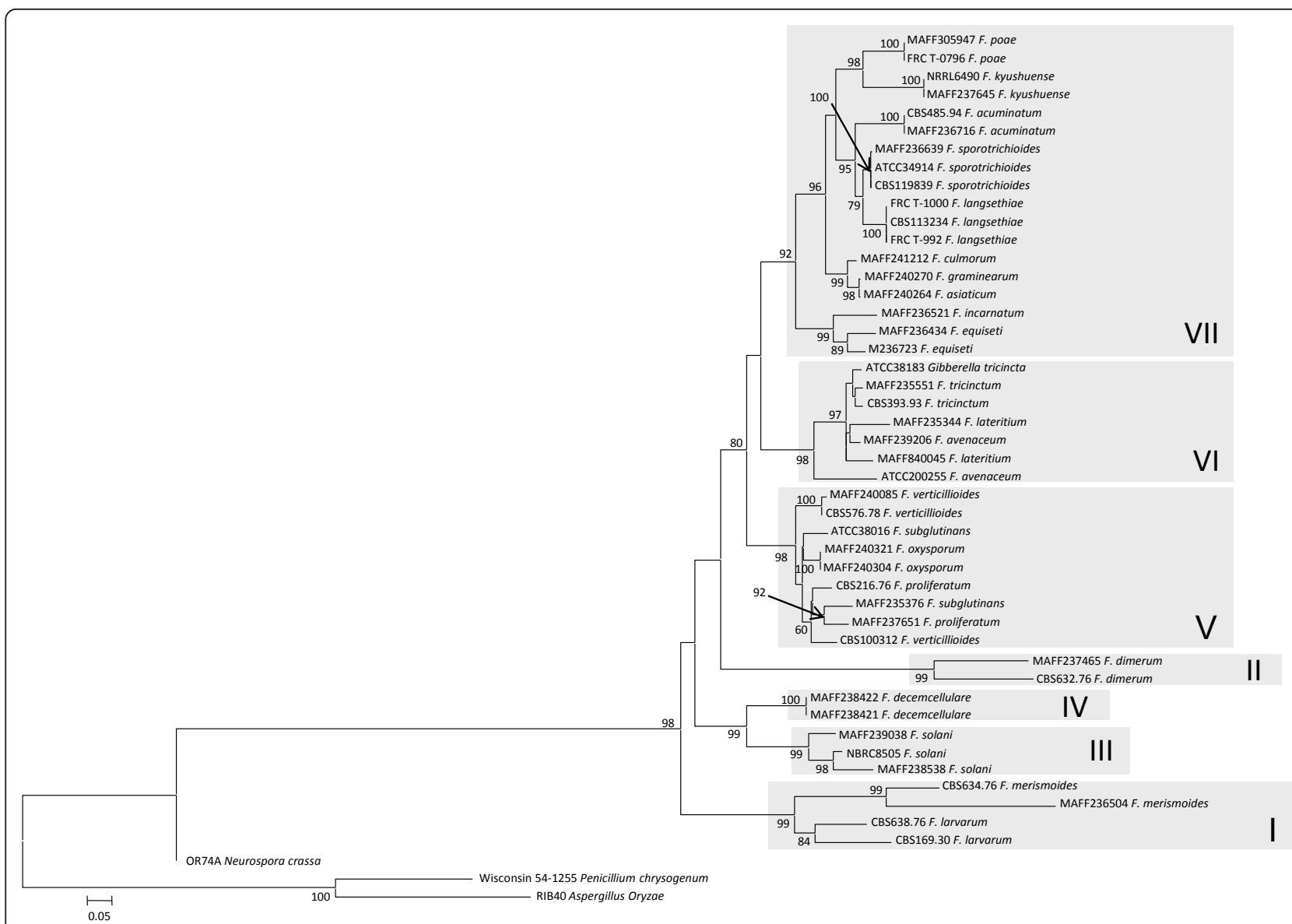

Figure 3 Maximum likelihood trees for the Fusarium genus and related genera inferred from $E F-1 \alpha$. The GTR $+1+\Gamma$ model was used as the model for nucleotide substitution. Branch lengths are proportional to the estimated number of nucleotide substitutions. Each codon position was analysed separately. The BP values over 75\% are displayed on the nodes (BP; 1000 replicates).

genes had a high resolution for the relationship among conspecific strains or closely related species in the same clade of I to VII (the lower taxa) (Figures 2, 3 and). However, in the cases of $\beta$-tub and EF-1 $\alpha$, the alignment of amino acid sequences among Fusarium species indicated that almost all of the substitutions were singletons, and parsimonious informative sites were limited (data not shown). Therefore, almost all of the substitutions which occurred within the genomes of Fusarium species were synonymous.

Pairwise comparisons of the substitution distances for the nucleotide and amino acid sequences are displayed in Figure 5 (Additional file 6). The distances in these graphs were divided into 4 groups; between conspecific Fusarium-strains, between Fusarium-strains of different species in the same clade of I to VII, between Fusariumstrains in different clades of I to VII, and between strains of Fusarium species and other genera. As for the nonsynonymous substitutions of $\beta$-tub and $E F-1 \alpha$, the numbers of non-synonymous substitutions in these genes were too few to reconstruct the phylogenetic relationships especially among clades I to VII and among Fusarim and its related genera (the higher taxa) in the lineage of the Fusarium genus (Figure 5A). The other 4 regions, namely, the rDNA cluster, the synonymous substitutions of $\beta$-tub and $E F-1 \alpha$, and the introns within $E F$ $1 \alpha$, displayed the graphs forms split up into 2 groups: the synonymous substitutions of $\beta$-tub/the introns within $E F-1 \alpha$ and the rDNA cluster/the synonymous substitutions of $E F-1 \alpha$ (Figure 5). The graphs of the former group (Figures $5 \mathrm{C}$ and $5 \mathrm{E}$ ) were almost parallel to the $x$ axis for the higher taxa. This implies that these 2 regions are completely saturated in the case of examining among the higher. However, these substitutions are useful for examining among the lower taxa. The graphs of the latter group (Figures 5B and 5D) are not linear but still everincreasing, and the substitution rates of this group are not so slow. This result suggests that these substitutions can provide information for phylogenetic reconstruction for both the higher and lower taxa.

One of the aims of this study was to provide a comprehensive description of the phylogenetic relationships 


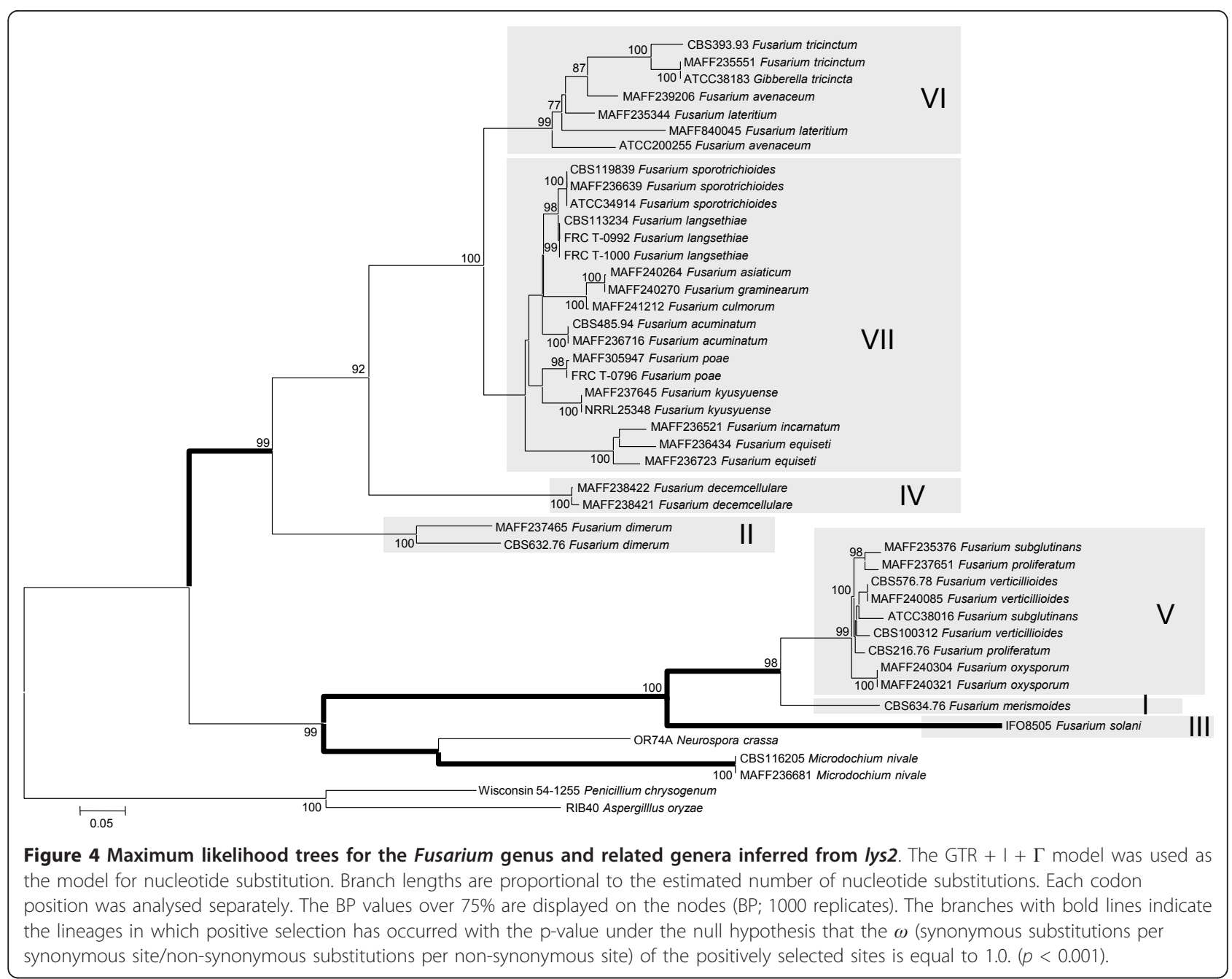

among Fusarium species and closely related species, including both the higher and lower taxa. Therefore, we require information obtained from multiple substitutions in multiple genes to reconstruct the phylogenetic tree. In this study, we considered all of the substitution information obtained from the rDNA cluster and the $\beta$-tub and $E F-1 \alpha$ genes. Substitutions within lys 2 were removed from our dataset because this locus is not suitable for the phylogenetic analysis of Fusarium and its related species. We had to modify the weighting of some nucleotides in the analysis because the information obtained from substitutions varied among sites with a partition model, as described in the materials and methods.

\section{Phylogenetic relationships among clades of the Fusarium genus}

The ML tree based on the combined data from the rDNA cluster and the $\beta$-tub and $E F-1 \alpha$ is displayed in Figure 6 . The BP values of more than $75 \%$ are presented

Table 1 The comparison of the tree topologies for relationships of clades I to VII based on each gene

\begin{tabular}{ccccc}
\hline & \multicolumn{4}{c}{ The difference of the log-likelihood score from ML tree \pm SD } \\
\cline { 2 - 5 } Topology & $\boldsymbol{\beta}$-tub & EF- $\boldsymbol{\alpha}$ & rDNA cluster & lys2 \\
\hline ML tree of/ $\beta$-tub & $<-4585.10>$ & $-1.67 \pm 1.81$ & $-9.62 \pm 5.69$ & $-571.47 \pm 40.65^{\mathrm{a}}$ \\
ML tree of EF-1 $\alpha$ & $-4.12 \pm 4.24$ & $<-8781.88>$ & $-7.28 \pm 4.93$ & $-512.43 \pm 43.93^{\mathrm{a}}$ \\
ML tree of rDNA cluster & $-16.34 \pm 7.73$ & $-19.78 \pm 8.29$ & $<-4965.10>$ & $-514.52 \pm 42.25^{\mathrm{a}}$ \\
ML tree of lys2 & $-33.97 \pm 12.45^{\mathrm{a}}$ & $-78.40 \pm 16.91^{\mathrm{a}}$ & $-91.24 \pm 19.33^{\mathrm{a}}$ & $<-9803.46>$ \\
\hline
\end{tabular}

Angled parenthes: the absolute value of the log likelihood score of the ML tree.

${ }^{\text {a }}$ Significantly smaller score of the log-likelihood than that of the ML tree by the SH test. 


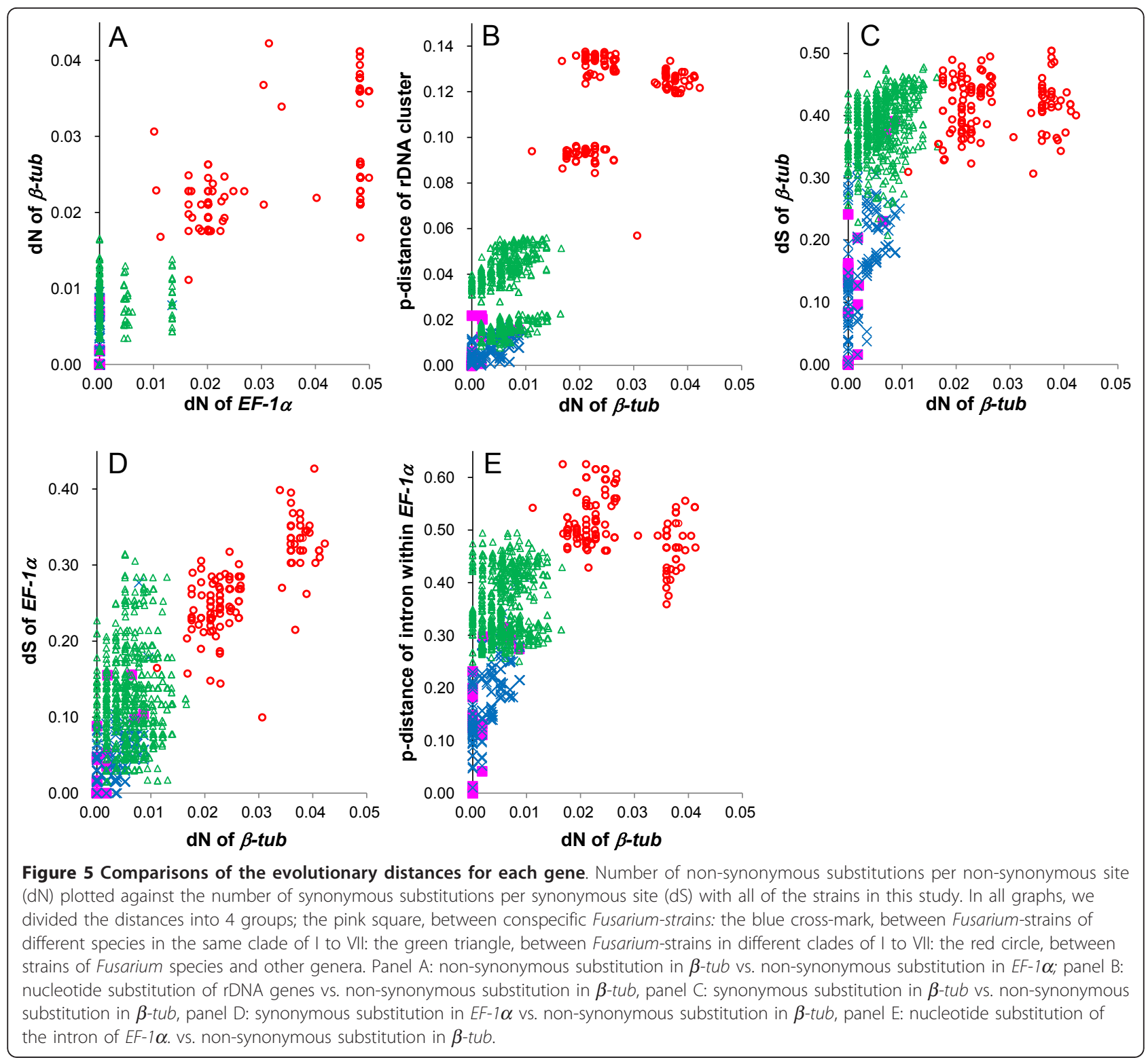

on the nodes. Our ML tree showed that $M$. nivale does not belong to any of the 7 major clades of the Fusarium species, and that this species, which is the common ancestor of Fusarium species and N. crassa, rapidly diverged during short term. Therefore, our analyses supported the previously described separation of M. nivale from the Fusarium genus [13,24].

Booth [5] proposed that closely related sections have the same teleomorph genus, and O'Donnell et al. [25] indicated that all Fusarium species with teleomorphs belonging to the Gibberella genus form a clade known as the Gibberella clade. In this study, all of the examined species with teleomorphs belonging to the Gibberella genus clustered in a super-clade consisting of clades
V to VII (Figure 6). Our results thus support the classification of the Gibberella clade.

Marasas et al. [1] and Pitt and Hocking [2] were reviewed that many Fusarium species produced many kinds of mycotoxins and these toxins are associated with human and animal health hazard. One of the main mycotoxins which naturally pollute agricultural crops, is trichotecenes or fumonisins. Our ML tree indicated that most Fusarium species which produce trichothecenes, clustered in a super-clade consisting of clade VI and VII (Figure 6). This relationship suggested that a common ancestor had acquired the capacity to produce trichothecenes, and then some species might lose this capacity. 


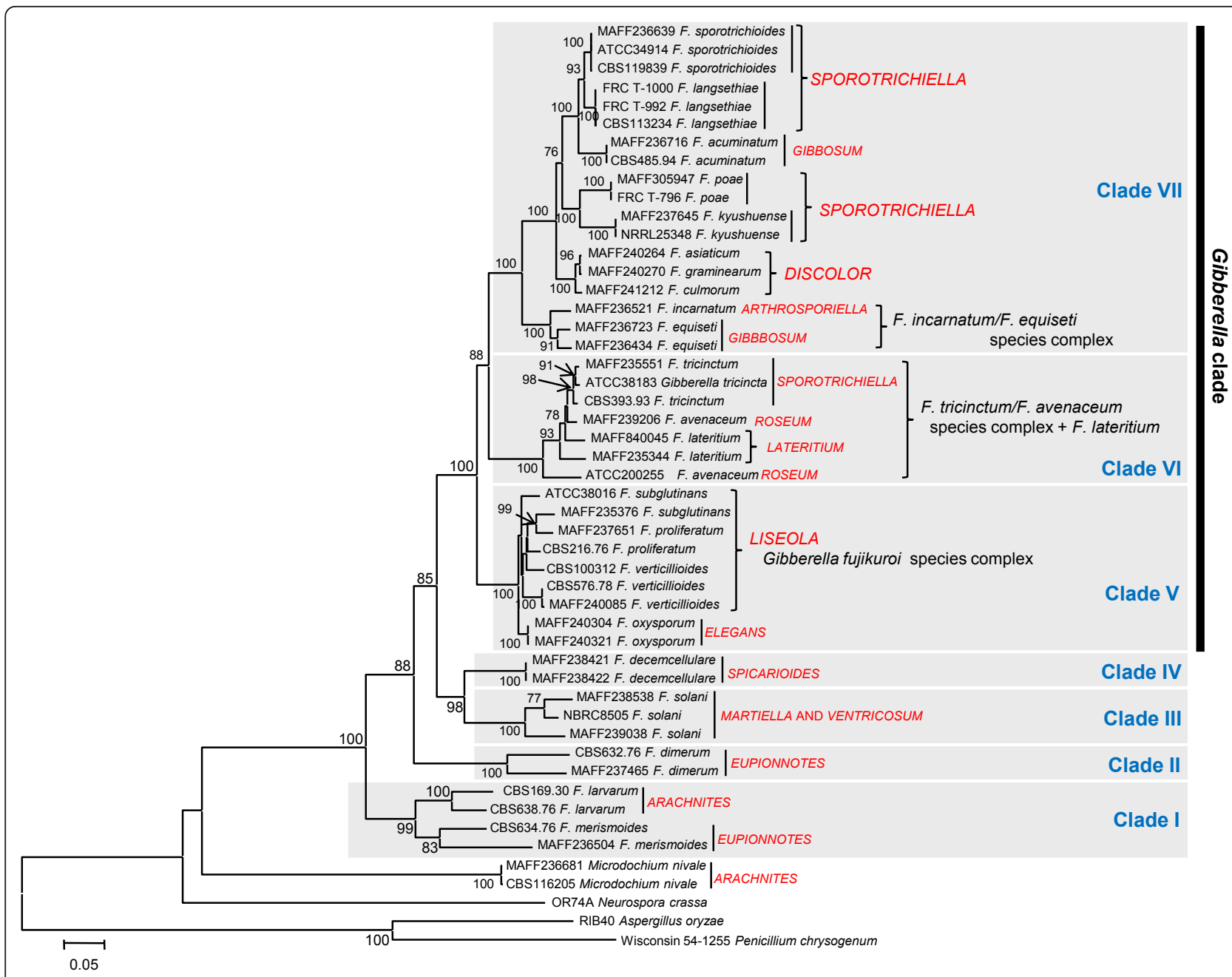

Figure 6 Maximum likelihood tree of the Fusarium genus and related genera inferred from the combined sequences of the rDNA cluster and the $\beta$-tub, and EF- $\alpha$ genes. Taking into account the different tempos and modes of nucleotide substitutions, all parameters of the substitution model were separately estimated for each gene using the GTR $+I+\Gamma$ model. The branch lengths are proportional to the estimated number of nucleotide substitutions. For the protein-coding genes $\beta$-tub and EF- $1 \alpha$, each codon position was analysed separately. The bootstrap probability (BP; 1000 replicates) values over 75\% are displayed on the nodes. Sections supported by morphological taxonomic systems are described, and include Arachnites, Arthrosporiella, Discolor; Elegans, Eupionnotes, Gibbosum, Lateritium, Liseola, Martiella and Ventricosum, Roseum, Spicarioides, and Sporotrichiella.

\section{Verification of monophyly of the sections and species of the Fusarium genus}

In our ML tree (Figure 6), many sections, which have previously been defined based upon morphological characteristics, were not verified as monophyletic. In this analysis, only Discolor (100\% BP) was supported by a BP value greater than $75 \%$. Sections other than Discolor, namely Arachnites, Eupionnotes, Gibbosum, and Sporotrichiella, which include 2 or more species in this study, formed the paraphyletic and polyphyletic groups. These results are consistent with previous studies reporting that taxonomic groups, which were traditionally classified using the morphological species concept, are not always recovered in molecular phylogenetic analyses of the Fusarium genus. In particular, in the Eupionnotes section, species were assembled using the morphological species concept with section-specific characteristics such as a very slow growth rate on potato dextrose agar (PDA; Eiken) a yeast-like appearance due to the absence of aerial mycelia, absence of microconidia, and small macroconidia generally having only 1 to 2 septa $[5,8]$. Our results indicated that these characteristics are not synapomorphies shared among only species in this section, such as F. dimerum and F. merismoides. Moreover, morphological characteristics such as slow growth rate, color of colonies on PDA from below (white to tan), absence of microconidia, and small macroconidia appear in the basal lineages containing species of clades I and 
II, and $M$. nivale. Therefore, we consider these characteristics to be ancestral, and that they remain in species of the basal lineages as symplesiomorphies. It is difficult to distinguish among the synapomorphies, the symplesiomorphies, and convergent derived characters. Species recognition on the basis of morphology often comprises several species that are recognized on the basis of molecular phylogeny.

Although the morphological species concept does not reflect the phylogenetic tree of the Fusarium genus, this does not imply that morphological characteristics are not useful for identification and taxonomy. On the contrary, species recognition based on morphological characteristics is also useful for identifying unknown species because morphological characteristics can be widely applied to any species, not only those of the Fusarium genus but also other fungi $[3,26]$. Fusarium isolates can be initially classified on the basis of morphological similarity, with the awareness that sections are in fact a means of artificial grouping. Thus, it is still necessary to use recognition methods based upon morphological characteristics in combination with the phylogenetic recognition method.

Although our ML tree was constructed with a high resolution, the relationships among species that together form a complex could not be resolved. Previous molecular phylogenetic analyses have suggested that a species of the Liseola section, defined by traditional taxonomy based on the morphological species concept, actually includes multiple species, and that the re-defined novel species, which are recognized mainly by mating types, constitute the Gibberella fujikuroi species complex [15-28]. The ML tree obtained in this study (Figure 6) demonstrates convoluted, nested structures of species within the Liseola section. The monophylies of F. subglutinans, F. proliferatum, and F. verticillioides, which have been recognized by the traditional morphological species concept, were not recovered. When we recognize species based on the novel taxonomic system (see Table 2: species re-identified by molecular method), our ML tree could not resolved the phylogenetic relationships among re-identified species in the "Gibberella fujikuroi species complex" excluding the sister relationship between F. phylophilum and F. fujikuroi. This difficulty of resolving phylogenetic relationships in this complex is probably caused by rapid divergence events occurred in this species complex. It is implied by the shortened internal branches in this species complex in our ML tree.

Furthermore, our study detected an additional intermingled, nested structure as a species complex containing the $F$. avenaceum/F. tricinctum/F. lateritium clade (clade VI in Figures 1 and 6). Regarding F. avenaceum, the affinities with $F$. acuminatum have been suggested by previous morphological and molecular studies $[8,28]$.
However, other molecular studies have suggested that $F$. avenaceum is more closely related to $F$. tricinctum than to $F$. acuminatum $[29,30]$. Moreover, the ML tree in this study suggested the presence of a $F$. avenaceum/F. tricinctum/F. lateritium clade (Figure 6). The phylogenetic hypothesis of a sister-species relationship between $F$. avenaceum and $F$. acuminatum was completely rejected by our ML tree in Figure 6. Further studies with more strains of each of the species within these complexes are required for the clarification of taxonomic ambiguities.

\section{Adaptive evolution of the lys 2 gene within the Fusarium genus}

The topology of the lys 2 tree was very different from that of the other trees (Figure 1, 2, 3, 4 and Table 1), and it indicated that the Fusarium genus is paraphyletic. Interestingly, by further investigation using the branchsite model, we detected many branches which displayed evidence of positive selection (The $\mathrm{p}$ value of the likelihood ratio test $<0.001$ ), indicated by bold branches in our lys 2 tree (Figure 4). Generally, the incongruence of the species trees and the species trees were caused by the following three reasons: (1) ancestral polymorphism and incomplete lineage sorting, (2) gene duplication, and (3) horizontal gene transfer. We briefly describe these three hypotheses respectively as well as the difficulties of them in the following paragraphs.

In support of the first hypothesis, there was the polymorphism in the ancestral population of the Fusarium genus and its related genera, and some alleles appeared to have been positively selected, and finally fixed in each lineage independently. However, we should assume that the ancestral polymorphism may have been maintained for a very long time, such as several hundred million years. For the $18 \mathrm{~S}$ rDNA gene, the average number of nucleotide substitutions between Fusarium and $M$. nivale is 17.0 and that between Fusarium and N. crassa is 30.9 . This is similar to the number of differences observed between the human and chicken $18 \mathrm{~S}$ rDNA genes (24 substitutions), and these lineages are thought to have diverged approximately 320 million years ago [31]. In the other hand, the average numbers of the nucleotide substitutions among Fusarium species is 1.9. The large difference among three genera and the small difference within the Fusarium genus indicate that it had taken long time until the emergence of the latest common ancestor of the Fusarium genus after the split of three genus.

The second hypothesis is that a gene duplication of lys 2 event may have occurred in the common ancestor of the Fusarium genus and its related genera. Therefore, the amino acid sequences of these groups could radically change. In each lineage, 1 copy may have been positively selected, while the other may have become a 
Table 2 Strains of the genus Fusarium and Fusarium-related spece is used in this study

\begin{tabular}{|c|c|c|c|c|}
\hline Section $^{a}$ & $\begin{array}{c}\text { Species registered in resorce } \\
\text { organization }\end{array}$ & $\begin{array}{l}\text { Species in tradittional } \\
\text { taxonomic system }\end{array}$ & $\begin{array}{l}\text { Species re-identificated by } \\
\text { molecular method }^{\mathrm{b}}\end{array}$ & Strain No. \\
\hline \multirow[t]{2}{*}{ Arachnites } & F. larvarum & F. larvarum & Not identified & $\begin{array}{c}\text { CBS }^{C} 169.30 \\
\text { CBS } 638.76 \text { (Isotype } \\
\text { strain) }\end{array}$ \\
\hline & Microdochium nivale & F. nivale & Not identified & $\begin{array}{c}\text { CBS } 116205 \text { (Isotype } \\
\text { strain) } \\
\text { MAFF }^{d} 236681\end{array}$ \\
\hline \multirow[t]{2}{*}{ Arthrosporiella } & F. incarnatum & F. semitectum & $\begin{array}{l}\text { F. incarnatum-equiseti species } \\
\text { complex }\end{array}$ & MAFF 236521 \\
\hline & F. culmorum & F. culmorum & F. cerealis & MAFF 241212 \\
\hline \multirow[t]{2}{*}{ Discolor } & F. asiaticum & F. graminearum & F. asiaticum & MAFF 240264 \\
\hline & F. graminearum & & F. graminearum & MAFF 240270 \\
\hline \multirow[t]{2}{*}{ Elegans } & F. oxysporum & F. oxysporum & F. oxysporum species complex & $\begin{array}{l}\text { MAFF } 240304 \\
\text { MAFF } 240321\end{array}$ \\
\hline & F. dimerum & F. dimerum & F. lunatum & $\begin{array}{l}\text { CBS } 632.76 \text { (Neotype } \\
\text { strain) }\end{array}$ \\
\hline \multirow[t]{2}{*}{ Eupionnotes } & & & F. penzigii & MAFF 237465 \\
\hline & F. merismoides & F. merismoides & Not identified & $\begin{array}{c}\text { CBS } 634.76 \text { (Type strain) } \\
\text { MAFF } 236504\end{array}$ \\
\hline \multirow[t]{2}{*}{ Gibbosum } & F. equiseti & F. equiseti & $\begin{array}{l}\text { F. incarnatum-equiseti species } \\
\text { complex }\end{array}$ & $\begin{array}{l}\text { MAFF } 236434 \\
\text { MAFF } 236723\end{array}$ \\
\hline & F. acuminatum & F. acuminatum & F. armeniacum & $\begin{array}{l}\text { CBS } 485.94 \\
\text { MAFF } 236716\end{array}$ \\
\hline \multirow[t]{4}{*}{ Lateritium } & F. lateritium & F. lateritium & F. lateritium & MAFF 235344 \\
\hline & & & Not identified & MAFF 840045 \\
\hline & F. proliferatum & F. proliferatum & F. phylophilum & CBS 216.76 (Type strain) \\
\hline & & & F. fujikuroi & MAFF 237651 \\
\hline \multirow[t]{5}{*}{ Liseola } & F. subglutinans & F. subglutinans & F. subglutinans & ATCC 38016 \\
\hline & & & F. sacchari & MAFF 235376 \\
\hline & & & F. verticillioides & $\begin{array}{c}\text { CBS } 576.78 \text { (Epitype } \\
\text { strain) }\end{array}$ \\
\hline & F. verticillioides & F. moniliforme & F. thapsinum & CBS 100312 \\
\hline & & & F. verticillioides & MAFF 240085 \\
\hline $\begin{array}{l}\text { Martiella- } \\
\text { Ventricosum }\end{array}$ & F. solani & F. solani & F. solani species complex & $\begin{array}{l}\text { MAFF } 238538 \\
\text { MAFF } 239038 \\
\text { NBRC }^{f} 8505\end{array}$ \\
\hline \multirow[t]{2}{*}{ Roseum } & F. avenaceum & F. avenaceum & F. nurragi & $\begin{array}{c}\text { ATCC } 200255 \text { (Type } \\
\text { strain) } \\
\end{array}$ \\
\hline & & & Not identified & MAFF 239206 \\
\hline \multirow[t]{3}{*}{ Spicarioides } & F. decemcellulare & F. decemcellulare & Not identified & $\begin{array}{l}\text { MAFF } 238421 \\
\text { MAFF } 238422\end{array}$ \\
\hline & F. kyushuense & Not described & F. kyushuense & $\begin{array}{c}\text { MAFF } 237645 \text { (Ex } \\
\text { holotype strain) } \\
\text { NRRL }^{9} 6490 \text { (Type } \\
\text { strain) }\end{array}$ \\
\hline & F. langsethiae & Not described & F. langsethiae & $\begin{array}{c}\text { CBS } 113234 \text { (Holotype } \\
\text { strain) } \\
\text { FRCh T-0992 } \\
\text { FRC T-1000 } \\
\end{array}$ \\
\hline \multirow[t]{2}{*}{ Sporotrichiella } & F. poae & F. poae & Not identified & $\begin{array}{l}\text { FRC T-0796 } \\
\text { MAFF } 305947\end{array}$ \\
\hline & F. sporotrichioides & F. sporotrichioides & Not identified & $\begin{array}{l}\text { ATCC } 34914 \\
\text { CBS } 119839 \\
\text { MAFF } 236639\end{array}$ \\
\hline
\end{tabular}


Table 2 Strains of the genus Fusarium and Fusarium-related spece is used in this study (Continued)

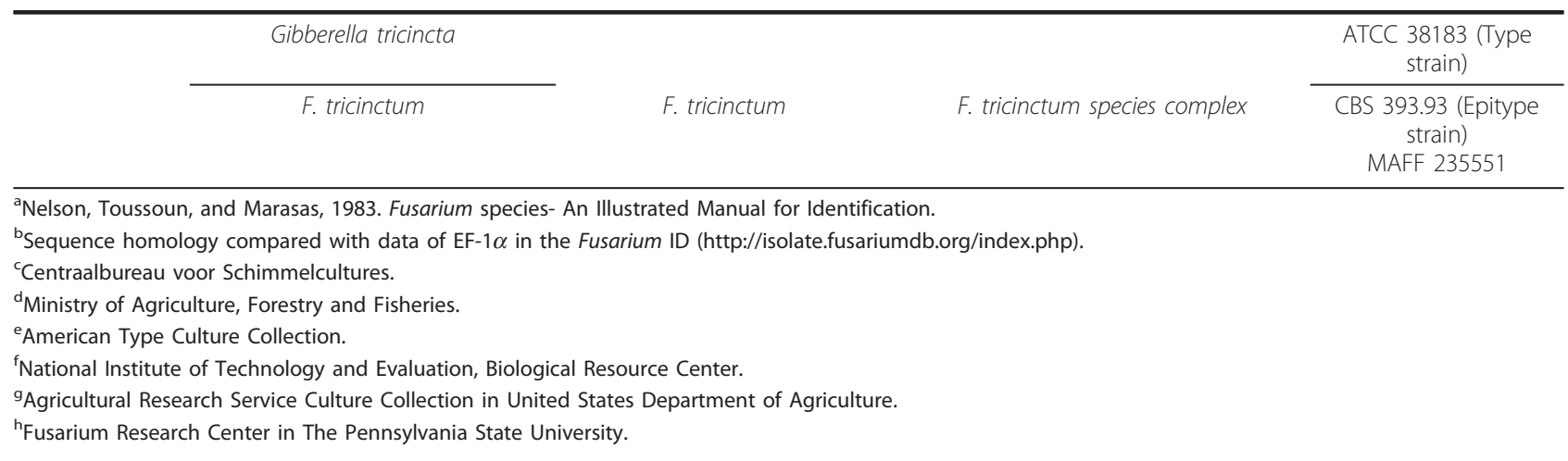

non-functional pseudogene which was eventually purged from the genome. We found additional lys 2 copies in the genomes of $F$. oxysporum and Nectria haematococca (anamorph; F. solani), but not in the genomes of $F$. verticillioides and Gibberella zeae (anamorph; F. graminearum), which were obtained from GenBank and the Fusarium Comparative Database (http://www.broadinstitute.org/annotation/genome/fusarium_group/MultiHome. html). We re-constructed the gene tree of lys 2 with sequences from this study and from GenBank (Figure 7). The tree suggests that gene duplications may occur repetitively. However according to our analysis using the branch model, the additional lys2 gene copies may still be subject to purifying selection (data not shown). Therefore, it is unlikely that 1 copy will be completely purged from the genome in each lineage after the alternative copy has been positively selected.

The third hypothesis is that horizontal gene transfer has repeatedly occurred from the genome of 1 lineage to another. Horizontal gene transfer in the fungal genome has previously been observed among various fungal species including $F$. oxysporum [11,32]. We can infer when and where such events might have occurred during the evolutionary history of lys 2 by comparing between the species tree (Figure 6) and the gene tree (Figure 4). The first horizontal gene transfer appears to have occurred from $N$. crassa (or M. nivale) to the Fusarium species in clade III. A second horizontal gene transfer appears to have occurred from clade III to clades I and V, respectively. The hypothesis that repetitive gene transfer events have occurred explains not only the positive selection but also the distantly related phylogenetic positions between the original lys 2 gene and the additional lys 2 copies found only in F. oxysporum and N. haematococca (Figure 7). However, the mechanism by which the new gene copy completely replaced the original copy in the hosts remains unclear.

Among these three hypotheses, the first hypothesis; ancestral polymorphism and incomplete lineage sorting, is unlikely. As mentioned above, although other two hypothesis can partially explain the incongruence of the gene trees and positive selections of lys2, the difficulties remain in all of the hypotheses. Hence, we cannot identify the reasons for them from our results.

The lys 2 gene is fungus specific and is related to the synthesis of lysine [15]. Our results indicate that this gene has been subjected to positive selection within the Fusarium genus. Therefore, the metabolism of lysine is expected to be similar among the species within clades I, III, and V and within clades II, IV, VI, and VII. However, this should be confirmed using biochemical experiments. An et al. [15,33] and Watanabe et al. [16] did not report multiple copies of lys 2 in the genomes of fungal genera such as Aspergillus, Byssochlamys, Saccharomyces, and others excluding Fusarium. Therefore, research and detection of other genera containing multiple copies of lys 2 in their genomes is required to understand the diversity of lysine-metabolizing systems of fungi. At the same time, we should note the difficulties of the estimation of the $\omega$ ratios (the number of nonsynonymous substitutions per non-synonymous site/the number of synonymous substitutions per synonymous site; $\mathrm{dN} / \mathrm{dS}$ ) in such a divergent taxon. The detection of the positive selection based on the $\omega$ ratios is highly sensitive to the saturation of the synonymous substituions. Since the relative evolutionary rate of the lys 2 is high among the genetic markers used in this study [34], it is possible that the synonymous substitutions of lys 2 were already saturated. However, the numbers of the multiple synonymous substitution at the same synonymous sites (multiple hits) were well estimated by the ML method using the codon substitution model, and ML method effectively correct the effect of multiple hits (Additional files 7 and 8). Moreover, w e applied the strict criterion to evaluate the statistical significance $(\mathrm{p}<0.001)$ to completely exclude the possibility of the overestimation of the detection for the positive selection. Therefore, we could completely exclude the possibility that some of the detected positive selections were falsepositive. 


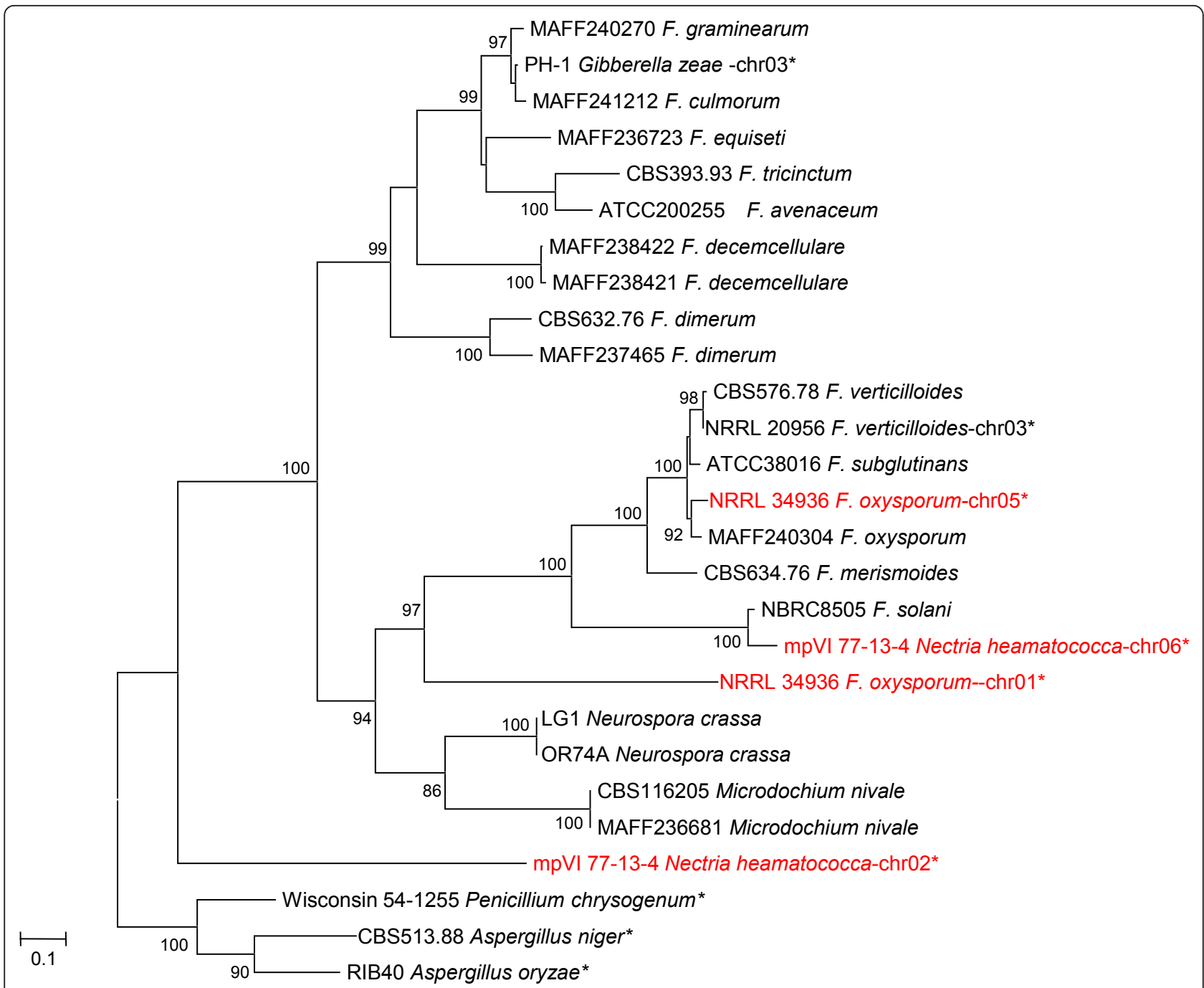

Figure 7 Maximum likelihood tree inferred from lys2. The GTR $+1+\Gamma$ model was used as the model of nucleotide substitution. The bootstrap probability (BP; 1000 replicated) values more than $75 \%$ are shown on the nodes. Branch lengths are proportional to the estimated number of nucleotide substitutions. The lys2 sequences of Wisconsin 54-1255 Penicillium chrysogenum (XM_002564615), CBS513.88 Aspergillus niger (XM_001401869), and RIB40 Aspergillus oryzae (XM_001820142) were used as outgroups. With the Fusarium dataset sequenced in this study, the lys2 sequences of PH-1 Gibberella zeae, NRRL 34936 Fusarium oxysporum, NRRL 20956 Fusarium verticillioides, mpVI 77-13-4 Nectria haematococca and Neurospora crassa were downloaded from the Fusarium Comparative Database, and also analysed. Data obtained from GenBank or were downloaded from the Fusarium Comparative Database are denoted by an asterisk. The species which have additional lys2 copies in their genomes are indicated in red.

Further understanding of the evolutionary processes of lys 2 and other genes is very important. To date, the full genome sequences of Fusarium are available for only 4 species of the Gibberella clade. The full genome sequences from other clades of Fusarium may elucidate the evolutionary processes of the genome, and such studies are currently in progress.

\section{Conclusion}

This study reports the reliable phylogenetic tree of the higher and lower taxonomy of the lineage of the Fusarium genus. Our ML tree clearly indicates that there are
7 major clades containing Fusarium species. These clades were supported with high BP values in all of the phylogenetic trees based on single genes. Moreover, our results indicate the considerable differences in the evolutionary histories of multiple genes in the lineage of the Fusarium genus, particularly lys 2.

\section{Methods}

Strains

The strains used in this study are listed in Table 2 . We selected 24 species from the genus Fusarium and its related genera according to nomenclature system 
proposed by Nelson et al. [8]. This nomenclature is based on traditional species recognition methods. It is very simple and systematized taxonomy, and is widespread application in the field of identification of Fusarium isolates. To cover a wide range of taxonomic groups, we selected additional species referring molecular phylogenetic studies [35,36]. Each species includes one to three strains, and we tested a total of 47 strains. We further purified all Fusarium strains by the singlespore method [8].

\section{DNA extraction}

We used two subcultures of each Fusarium and Fusarium-related species obtained by the single-spore method for sequencing. We checked their sequence identity between two subcultures to confirm purity of the strain. Samples were cultured on synthetic low nutrient agar (SNA; [26]) slant media supplemented with chloramphenicol $100 \mathrm{mg} / \mathrm{l})$ at $25^{\circ} \mathrm{C}$ for 14 days. Mycelia and conidia from the slant culture were inoculated into $1 \mathrm{ml}$ potato dextrose broth (Difco Laboratories) in a microtube, and were incubated at $25^{\circ} \mathrm{C}$ for 3 days. These fungal bodies were clumped by centrifugation at $18,000 \times g$ for $10 \mathrm{~min}$ in a microtube. The genomic DNA was extracted from these pellets using the SDS method with minor modifications, as previously described [37].

\section{Amplification and sequencing of genes}

The ribosomal RNA gene (rDNA) cluster region, including the 3' end of the $18 \mathrm{~S}$ rDNA, the internal transcribed spacer region 1 (ITS1), the $5.8 \mathrm{~S} \mathrm{rDNA}$, and the $5^{\prime}$ end of the $28 \mathrm{~S} \mathrm{rDNA}$, and the $\beta-t u b, E F-1 \alpha$, and lys 2 were selected as the regions for analysis. We performed amplification reactions with the primer pair for each gene using TaKaRa ExTaq (TaKaRa Bio Inc.), according to the manufacturer's instructions, in a thermal cycler (GeneAmp PCR System 9700; Applied Biosystems). The ITS1, 5.8S rDNA, and 5 ' end of the 28S rDNA were amplified in 1 fragment using the primer pair, ITS5 (5'-GGAAGTAAAAGTCGTAACAAGG-3'; [38])/NL4 (5'-GGTCCGTGTTTCAAGACGG-3'; [36]). For PCR amplification other than that for ITS1, 5.8S rDNA, and 28S rDNA, we used the following primer pairs: FF1 (5'-GTTAAAAAGCTCGTAGTTGAAC-3'; [39])/FR1 (5'-CTCTCAATCTGTCAATCC TTATT-3'; [39]) for 18S rDNA; Btu-F-F01 (5'-CAGACC GGTCAGTGCGTAA-3')/Btu-F-R01 (5'-TTGGGGTCG AACATCTGCT-3') for $\beta$-tub; EF-1 (5'-ATGGGTAAGGARGACAAGAC-3'; [40])/EF-2 (5'-GGARGTACCAGTSATCATGTT-3'; [40]) for $E F-1 \alpha$; and 2 primer pairs of Fulys2-F03mix (5'-CTTTGTTGGTGATGTTCTSA-3')/ Fulys2-R01 (5'-TGGTAGGTCCGATATCGGT-3') and Fulys2-F04mix (5'- GCYATGGGDCARATYCTKGT -3')/ Fulys2-R04mix (5'-CGGYTCYTCRTTRCGRTCTCT-3') for lys 2 . The primer pairs for $\beta$-tub and lys 2 were designed based on sequences derived from primers used in previous studies, respectively $[16,17]$. They were designed to amplify the genes effectively. The PCR program consisted of an initial denaturing step at $94^{\circ} \mathrm{C}$ for $5 \mathrm{~min}, 35$ amplification cycles, and an additional extending step at $72^{\circ} \mathrm{C}$ for 3 min. For the primer pairs FF1/FR1, ITS5/NL4, Fulys2F03mix/Fulys2-R01, and Fulys2-F04mix/Fulys2-R04mix, the amplification cycles were $94^{\circ} \mathrm{C}$ for $30 \mathrm{~s}, 52^{\circ} \mathrm{C}$ for $40 \mathrm{~s}$, and $72^{\circ} \mathrm{C}$ for $1 \mathrm{~min}$ and $10 \mathrm{~s}$. For the primer pair Btu-FF01/Btu-F-R01, the amplification cycles were $94^{\circ} \mathrm{C}$ for $30 \mathrm{~s}, 60^{\circ} \mathrm{C}$ for $40 \mathrm{~s}$, and $72^{\circ} \mathrm{C}$ for $1 \mathrm{~min}$. The PCR products were purified using ExoSap-IT (USB; Cleveland, OH). Dye labelling of the PCR products was performed with the same primers which were used for each gene in the amplification reactions, using the BigDye Terminator v. 3.1 Cycle Sequencing Kit (Applied Biosystems). For the PCR products from ITS5/NL4, we used the additional primer ITS3 (5'-GCATCGATGAAGAACGCAGC-3'; [38]). The labelled PCR products were ethanol precipitated according to the manufacturer's instructions, and then directly sequenced using the ABI 3130 analyzer (Applied Biosystems). The sequences were assembled using ATGC software (Genetyx Corporation). The lys2 gene of strains CBS 169.30, CBS 638.76, MAFF 236504, MAFF238538, and MAFF 239038, and the EF-1 $\alpha$ gene of strains CBS 116205 and MAFF 236681 could not be amplified by PCR. The sequences determined in this study have been deposited in GenBank (accession nos. AA0000).

\section{Phylogenetic analysis based on DNA sequences}

The nucleotide sequence datasets for each gene (18S rDNA, ITS1, 5.8S rDNA, 28S rDNA, $\beta$-tub, EF- $1 \alpha$, and lys2) were automatically aligned using the MUSCULE program [41]. Alignments were carefully checked visually and were manually modified; all ambiguous sections were excluded from the analysis. All intron regions of $\beta$-tub and lys 2 were excluded, and we aligned only the exons. However, the introns for $E F-1 \alpha$ comprised a relatively large proportion of our sequence data for this gene (63.8\%), and these sections were retained for the analysis. We used $\beta$-tub, EF-1 $\alpha$, and lys 2 exon sequences from several Fusarium and its related genus species, including PH-1 Gibberella zeae, NRRL 34936 F. oxysporum, and NRRL 20956 $F$. verticillioides, as references for the alignments. These sequences were downloaded from the Fusarium Comparative Database. The final lengths of the sequences are as follows: rDNA cluster (1314 bp: $18 \mathrm{~S}$ rDNA $=509 \mathrm{bp}$, ITS1 $=101 \mathrm{bp}, 5.8 \mathrm{~S} \mathrm{rDNA}=159 \mathrm{bp}$, and $28 \mathrm{~S} \mathrm{rDNA}=545 \mathrm{bp})$, $\beta$-tub (768 bp), EF-1a (804 bp), and lys2 (948 bp). Two Eurotiomycetes species, Penicillium chrysogenum Wisconsin 54-1255 (GenBank accession nos. XM_002559715, XM_002564615, and AM920418) and Aspergillus oryzae RIB40 (GenBank accession nos. XM_001825624, XM_001820142, and AP007172) were used as outgroups. 
The Sordariomycetes species N. crassa OR74A (GenBank accession nos. XM_952576, XM_960303 and FJ360521) was also used in the analysis to examine the monophyly of the Fusarium genus.

Phylogenetic trees were inferred using the ML method. This method has the corrective effect of multiple hits. The RAxML program ver. 7.0.3 [42] was used for the heuristic search.

Taking into account the different tempos and modes of the nucleotide substitutions as described in Figure 5, we separately estimated all parameters using the GTR $+I+\Gamma$ substitution model for each partition. The partitions were as follows: rDNA region, ITS1 region, each codon site of $\beta$-tub, each codon site of lys 2 , introns of $E F-1 \alpha$, and each codon site of $E F-1 \alpha$. The branch lengths of each partition were estimated separately with independent model (see [43]). Since the RAxML program could not accurately estimate the branch lengths of $\beta$-tub by this method, we used the default option for this gene. To determine the confidence for the internal nodes, the rapid bootstrap method [42] was applied (1000 replications). The BASEML programs of PAML ver. 4.4 [44] were used for the exhaustive search. The GTR $+\Gamma$ model was used, and all parameters of the substation model were separately estimated using the same partitions as mentioned above. The branch lengths were estimated using the proportional model [45]. To evaluate incongruence among the different gene trees, we compared the log-likelihood scores and their standard deviations by the Shimodaira-Hasegawa SH test [46], using the BASEML program. As we only focused on the relationships among major clades (seven clades of the Fusarium genus, $M$. nivale and $N$. crassa), we swapped the major clades of the ML trees for each gene one by one, and then compared the different tree topologies.

We compared the observed substitutions among genes, except for lys2, to evaluate the effects of saturation due to multiple hits. Pairwise comparisons of the observed number of synonymous and non-synonymous substitutions per site were calculated for all sequences of the 50 tested strains by Nei and Gojobori's method [47], without any correction, using MEGA ver. 5.0 software [48]. The p-distances of the rDNA cluster and the EF-1 $\alpha$ introns were also calculated using MEGA ver. 5.0.

A $\chi^{2}$ test was used to examine nucleotide and amino acid composition bias in particular lineages using TREE-PUZZLE ver 5.2 [49]. Composition bias was tested with datasets containing only the $3^{\text {rd }}$ codon position sites, the combined $1^{\text {st }}$ and $2^{\text {nd }}$ codon position sites, and the combined $1^{\text {st }}, 2^{\text {nd }}$, and $3^{\text {rd }}$ codon position sites and amino acids.

\section{Detection of positive selection}

Positive selection in particular lineages was detected by the branch-site model [50] using the CODEML program of PAML ver. 4.4. The statistical significance of positive selection was tested using likelihood ratio tests to compare the observed substitutions with the null hypothesis, which assumed that the ratio of non-synonymous rates/ synonymous rates $(\omega)$ was equal to 1 [48].

\section{Additional material}

Additional file 1: Supplementary method S1.
Additional file 2: Supplementary figure S1. Maximum likelihood trees
of the genus Fusarium and its related genera inferred from 185 rRNA
gene (rDNA).
Additional file 3: Supplementary figure S2. Maximum likelihood trees
of the genus Fusarium and its related genera inferred from internal
transcribed spacer region 1.
Additional file 4: Supplementary figure S3. Maximum likelihood trees
of the genus Fusarium and its related genera inferred from 5.8S rDNA.
Additional file 5: Supplementary figure S4. Maximum likelihood trees
of the genus Fusarium and its related genera inferred from 285 rDNA.
The GTR + I + $\Gamma$ model was used as the model of the nucleotide
substitution. The nodal numbers indicate the bootstrap probability (BP;
1000 replicated). The branch lengths are proportional to the estimated
number of nucleotide substitutions. The BP values more than $75 \%$ are
shown on the nodes. Although the RAxML program inferred the ML tree
of 285 rDNA including all 50 strains, it could not be summarized nodal
BPs. Therefore, all identical sequences were excluded and remaining 31
sequences were used for the estimation of nodal BPs.
Additional file 6: Supplementary method S2.
Additional file 7: Supplementary method S3.
Additional file 8: Supplementary figure S5. Comparisons of the
evolutionary distances by two methods. Number of non-synonymous
substitutions per non-per synonymous site (dS). Panel A: Distance
method modified Nei-Gojorbori(JC) k = 2.5; panel B: ML method the
codon model (pairwise). The estimated synonymous substitution reached
plateau from the analysis by Nei Gojobori method. However there was
no such tendency from the analysis by the maximum likelihood method.
It means that although there were many multiple substitutions at the
synonymmous sites, the maximum likelihood effectively correct the
numbers of the multiple substitutions.

\section{Acknowledgements}

We wish to express our special thanks to Prof. Masami Hasegawa for his helpful comments and technical support. This study was partly supported by a grant from the Ministry of Health, Labour and Welfare of Japan, and the Cooperative Research Program of Medical Mycology Research Center, Chiba University (09-14).

\section{Author details}

'Division of Microbiology, National Institute of Health Sciences, Kamiyoga 118-1, Setagaya-ku, Tokyo 158-8501, Japan. ${ }^{2}$ School of Life Sciences, Fudan University, 220, Handan Rd. 200433, Shanghai, China. ${ }^{3}$ Veterinary Medical Science, Graduate School of Agricultural and Life Sciences, The University of Tokyo, Yayoi 1-1-1, Bunkyo-ku, Tokyo 113-8657, Japan. ${ }^{4}$ Food Research Laboratories, Mitsui Norin Co., Ltd., Miyabara 223-1, Fujieda-shi, Shizuoka 4260133, Japan.

\section{Authors' contributions}

MW, GK, and YH-K conceived and designed the study. MW, YS-K, and YH-K collected the fungal strains. MW and K-IL performed the experiments. TY analyzed the data. MW, TY, and YH-K drafted manuscript and wrote the paper. SK, YS-K, K-IL and GK participated in the preparing the draft and revised the manuscript. All authors read and approved the final manuscript.

Received: 31 March 2011 Accepted: 3 November 2011

Published: 3 November 2011 


\section{References}

1. Marasas WFO, Nelson PE, Toussoun TA: Toxigenic Fusarium Species. University Park: The Pennsylvania State University Press; 1984.

2. Pitt Jl, Hocking AD: Fungi and food spoilage. Berlin: Springer; 32009.

3. Taylor JW, Jacobson DJ, Kroken S, Kasuga T, Geiser DM, Hibbett DS, Fisher MC: Phylogenetic species recognition and species concepts in fungi. Fungal genetics and biology 2000, 31(1):21-32.

4. Kirk PM, Cannon PF, Minter DW, Stalpers JA, (eds.): Dictionary of the Fungi. Wallingford: CAB international; 102008.

5. Booth C: The genus Fusarium. Surrey: Commonwealth Mycological Institute; 1971.

6. Gerlach W, Nirenberg H: The Genus Fusarium: A Pictorial Atlas. Berlin: P. Parey; 1982.

7. Joffe AZ: A modern system of Fusarium taxonomy. Mycopathologia et mycologia applicata 1974, 53(1):201-228

8. Nelson PE, Toussoun TA, Marasas WFO: Fusarium species: An illustrated manual for identification. Univercity Park: The Pennsylvania State University Press; 1983.

9. Snyder WC, Hansen HN: The species concept in Fusarium. American Journal of Botany 1940, 27:64-67.

10. Wollenweber HW, Reinking OA: Fusarien, Ihre Beschreibung, Schadwirkung Und Bekampfung. Berlin: Paul Parey; 1935.

11. Marcet-Houben M, Gabaldón T: Acquisition of prokaryotic genes by fungal genomes. Trends in Genetics 2010, 26(1):5-8.

12. O'Donnell K, Cigelnik E, Nirenberg HI: Molecular systematics and phylogeography of the Gibberella fujikuroi species complex of Fusarium Mycologia 1998, 90:465-493.

13. O'Donnell K, Cigelnik E: Two divergent intragenomic rDNA ITS2 types within a monophyletic lineage of the fungus Fusarium are nonorthologous. Molecular phylogenetics and evolution 1997 7(1):103-116.

14. Azor M, Gene J, Cano J, Manikandan P, Venkatapathy N, Guarro J: Lessfrequent Fusarium species of clinical interest: correlation between morphological and molecular identification and antifungal susceptibility. Journal of Clinical Microbiology 2009, 47(5):1463-1468

15. Guadet J, Julien J, Lafay JF, Brygoo Y: Phylogeny of some Fusarium species, as determined by large-subunit rRNA sequence comparison. Molecular Biology and Evolution 1989, 6(3):227-242.

16. An KD, Nishida $H$, Miura $Y$, Yokota A: Aminoadipate reductase gene: a new fungal-specific gene for comparative evolutionary analyses. $B M C$ Evolutionary Biology 2002, 2(6)

17. Watanabe M, Kato Y, Togami K, Yamanaka M, Wakabayashi K, Ogawa H, Wasada Y, Goto K, Hara-Kudo Y, Amano N, et al: Evaluation of Gene Index for Identification of Byssochlamys spp. Journal of the Food Hygienic Society of Japan 2008, 49(2):82-87.

18. Zhong B, Yonezawa $T$, Zhong $Y$, Hasegawa M: The position of gnetales among seed plants: overcoming pitfalls of chloroplast phylogenomics. Molecular biology and evolution 2010, 27(12):2855-2863.

19. Nikaido M, Cao Y, Harada M, Okada N, Hasegawa M: Mitochondrial phylogeny of hedgehogs and monophyly of Eulipotyphla. Molecular phylogenetics and evolution 2003, 28(2):276-284.

20. Yonezawa $\mathrm{T}$, Hasegawa M: Was the universal common ancestry proved? Nature 2010, 468(7326):E9, discussion E10.

21. Kimura M: The neutral theory of molecular evolution. Cambridge: Cambridge University Press; 1983

22. Adachi J, Hasegawa M: MOLPHY Version 2.3: Programs for molecular phylogenetics based on maximum likelihood. Computer Science Monographs Tokyo: Institute of Statistical Mathematics; 1996, 1-150, vol 28.

23. Liu Y, Cotton JA, Shen B, Han X, Rossiter SJ, Zhang S: Convergent sequence evolution between echolocating bats and dolphins. Current Biology 2010, 20(2):R53-54.

24. Samuels GJ, Hallett IC: Microdochium stoveri and Monographella stoveri, new combinations for Fusarium stoveri and Micronectriella stoveri. Transactions of the British Mycological Society 1983, 81(3):473-483.

25. O'Donnell K, Sarver BA, Brandt M, Chang DC, Noble-Wang J, Park BJ, Sutton DA, Benjamin L, Lindsley M, Padhye A, et al: Phylogenetic diversity and microsphere array-based genotyping of human pathogenic Fusaria, including isolates from the multistate contact lens-associated U.S. keratitis outbreaks of 2005 and 2006. Journal of clinical microbiology 2007, 45(7):2235-2248
26. Nirenberg HI, Aoki T: Fusarium nisikadoi, a new species from Japan. Mycoscience 1997, 38:329-333.

27. Steenkamp ET, Wingfield BD, Coutinho TA, Zeller KA, Wingfield MJ, Marasas WF, Leslie JF: PCR-based identification of MAT-1 and MAT-2 in the Gibberella fujikuroi species complex. Applied and environmental microbiology 2000, 66(10):4378-4382.

28. Leslie JF, Summerell BA: The Fusarium Laboratory Manual. Blackwell ${ }^{\prime} 12006$.

29. Kristensen $R$, Torp M, Kosiak B, Holst-Jensen A: Phylogeny and toxigenic potential is correlated in Fusarium species as revealed by partial translation elongation factor 1 alpha gene sequences. Mycological research 2005, 109(Pt 2):173-186.

30. Turner AS, Lees AK, Rezanoor HN, Nicholson P: Refinement of PCRdetection of Fusarium avenaceum and evidence from DNA marker studies for phenetic relatedness to Fusarium tricinctum. Plant Pathology 1998, 47:278-288

31. Benton MJ, Donoghue PC: Paleontological evidence to date the tree of life. Molecular Biology and Evolution 2007, 24(1):26-53.

32. Ma $\sqcup$, van der Does HC, Borkovich KA, Coleman JJ, Daboussi MJ, Di Pietro A, Dufresne M, Freitag M, Grabherr M, Henrissat B, et al: Comparative genomics reveals mobile pathogenicity chromosomes in Fusarium. Nature 2010, 464(7287):367-373

33. An KD, Nishida H, Miura Y, Yokota A: Molecular evolution of adenylating domain of aminoadipate reductase. BMC Evolutionary Biology 2003, 3:9.

34. Watanabe M, Yonezawa T, Lee K, Kumagai S, Sugita-Konishi Y, Goto K, HaraKudo Y: Evaluation of genetic markers for identifying isolates of the species of the genus Fusarium. Journal of Sciences of Food and Agriculture 2011, 91(13):2500-2504.

35. Aoki T, O'Donnell K: Fusarium kyushuense, sp. now. from Japan. Mycoscience 1998, 39:1-6.

36. O'Donnell K: Fusarium and its near relatives. In The Fungal Holomorph: Mitoic, Meiotic and Pleomorphic Speciation in Fungal Systematics. Edited by: Reynolds DR, Taylor JW. Wallingford: CAB International; 1993:225-233.

37. Watanabe M, Lee K, Goto K, Kumagai S, Sugita-Konishi Y, Hara-Kudo Y: Rapid and effective DNA extraction method with bead grinding for a large amount of fungal DNA. Journal of food protection 2010, 73(6):1077-1084

38. White TJ, Bruns T, Lee S, Taylor J: Amplification and direct sequencing of fungal ribosomal RNA genes for phylogenetics. In PCR Protocols: A Guide to Methods and Applications. Edited by: Innis MA, Gelfand DH, Sninsky JJ. White TJ: Academic Press; 1990:315-322

39. Zhou G, Whong WZ, Ong T, Chen B: Development of a fungus-specific PCR assay for detecting low-level fungi in an indoor environment. Molecular and Cellular Probes 2000, 14(6):339-348.

40. O'Donnell K, Kistler HC, Cigelnik E, Ploetz RC: Multiple evolutionary origins of the fungus causing Panama disease of banana: concordant evidence from nuclear and mitochondrial gene sgenealogies. Proc Natl Acad SC USA 1998, 95(5):2044-2049.

41. Edgar RC: MUSCLE: a multiple sequence alignment method with reduced time and space complexity. BMC Bioinformatics 2004, 5:113.

42. Stamatakis $A$, Hoover $P$, Rougemont J: A rapid bootstrap algorithm for the RAxML Web servers. Systematic biology 2008, 57(5):758-771

43. Pupko T, Huchon D, Cao Y, Okada N, Hasegawa M: Combining multiple data sets in a likelihood analysis: which models are the best? Mol Biol Evol 2002, 19(12):2294-2307.

44. Yang Z: PAML 4: phylogenetic analysis by maximum likelihood. Mol Biol Evol 2007, 24(8):1586-1591.

45. Yang Z: Maximum-Likelihood Models for Combined Analyses of Multiple Sequence Data. Journal of Molecular Evolution 1996, 42(5):587-596.

46. Shimodaira H, Hasegawa M: Multiple Comparisons of Log-Likelihoods with Applications to Phylogenetic Inference. Molecular Phylogenetics and Evolution 1999, 16(8):1114-1116.

47. Nei M, Gojobori T: Simple methods for estimating the numbers of synonymous and nonsynonymous nucleotide substitutions. Molecular Biology and Evolution 1986, 3(5):418-426.

48. Tamura K, Dudley J, Nei M, Kumar S: MEGA4: Molecular Evolutionary Genetics Analysis (MEGA) software version 4.0. Molecular biology and evolution 2007, 24(8):1596-1599.

49. Schmidt HA, Strimmer $K$, Vingron M, von Haeseler A: TREE-PUZZLE: maximum likelihood phylogenetic analysis using quartets and parallel computing. Bioinformatics 2002, 18(3):502-504 
50. Yang Z, Wong WS, Nielsen R: Bayes empirical bayes inference of amino acid sites under positive selection. Molecular biology and evolution 2005, 22(4):1107-1118.

doi:10.1186/1471-2148-11-322

Cite this article as: Watanabe et al:: Molecular phylogeny of the higher and lower taxonomy of the Fusarium genus and differences in the evolutionary histories of multiple genes. BMC Evolutionary Biology 2011 11:322.

Submit your next manuscript to BioMed Central and take full advantage of:

- Convenient online submission

- Thorough peer review

- No space constraints or color figure charges

- Immediate publication on acceptance

- Inclusion in PubMed, CAS, Scopus and Google Scholar

- Research which is freely available for redistribution 